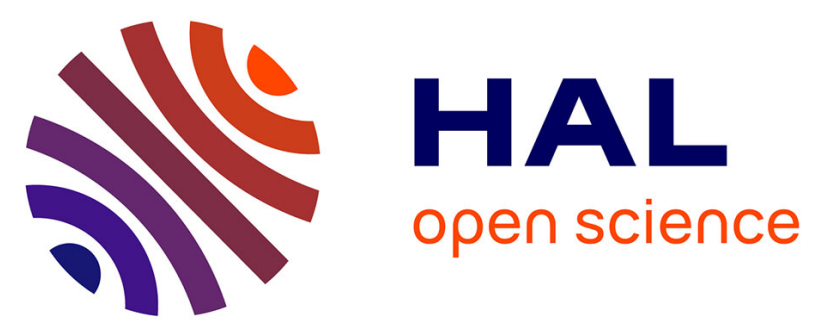

\title{
Conseiller le politique: des évaluations commanditées à la prospective en éducation. L'évolution des relations entre la recherche et le politique en Communauté française de Belgique
}

Aubert Angeline, Marc Demeuse, Antoine Derobertmasure, Nathanaël Friant

\section{To cite this version:}

Aubert Angeline, Marc Demeuse, Antoine Derobertmasure, Nathanaël Friant. Conseiller le politique: des évaluations commanditées à la prospective en éducation. L'évolution des relations entre la recherche et le politique en Communauté française de Belgique. Les Dossiers des sciences de l'éducation , 2007, 18, pp.121-130. halshs-00850149

\section{HAL Id: halshs-00850149 \\ https://shs.hal.science/halshs-00850149}

Submitted on 4 Aug 2013

HAL is a multi-disciplinary open access archive for the deposit and dissemination of scientific research documents, whether they are published or not. The documents may come from teaching and research institutions in France or abroad, or from public or private research centers.
L'archive ouverte pluridisciplinaire HAL, est destinée au dépôt et à la diffusion de documents scientifiques de niveau recherche, publiés ou non, émanant des établissements d'enseignement et de recherche français ou étrangers, des laboratoires publics ou privés. 


\section{Conseiller le politique : des évaluations commanditées à la prospective en éducation.}

\section{L'évolution des relations entre la recherche et le politique en Communauté française de Belgique.}

Angeline Aubert-Lotarski, Marc Demeuse, Antoine Derobertmasure et Nathanaël Friant Institut d'Administration scolaire, Université de Mons-Hainaut (Belgique)

L'évolution de la recherche en éducation en Communauté française de Belgique, notamment dans une fonction de conseil au politique, peut être analysée au regard du développement du champ de l'administration scolaire. Nous distinguons et illustrons par des exemples trois périodes qui se sont succédées depuis les années 1960, correspondant à trois objectifs des évaluations menées en réponse à des commandes : observer et décrire, supporter la mise en œuvre, et tracer des perspectives. En conclusion, nous interrogeons la posture épistémologique du chercheur au regard des notions de service public, de légitimité scientifique et de décision politique.

The evolution of research in education in the French Community of Belgium, in particular in its function of consultancy for the politics, can be analyzed regarding the development of school administration field. We distinguish and exemplify three successive periods since the 1960's. They match with three goals of evaluations responding to commissioned researches: to observe and describe, to support implementations, and to draw prospects. In conclusion, we question the epistemological posture of researchers in respect of the concepts of service of general interest, scientific legitimacy and political decision.

Mots-clefs: évaluation, politique, administration scolaire, prospective éducative, épistémologie, conseil, méthodologie, recherche en éducation 


\section{Conseiller le politique : des évaluations commanditées à la prospective en éducation.}

\section{L'évolution des relations entre la recherche et le politique en Communauté française de Belgique.}

Angeline Aubert-Lotarski, Marc Demeuse, Antoine Derobertmasure et Nathanaël Friant Institut d'Administration scolaire, Université de Mons-Hainaut (Belgique)

\section{Introduction}

Les relations entre universités et administration de l'enseignement, en Belgique, sont de nature particulière. La lutte qui a prévalu dès la naissance de l'Etat (Van Haecht, 2004), à la suite de la révolution de 1830 , entre le parti catholique et les libéraux a conduit à la mise en place d'une administration aussi faible que possible, permettant ainsi à l'enseignement privé confessionnel de se développer. On peut encore lire aujourd'hui, dans les structures et le fonctionnement du système éducatif, les traces de cette lutte. Les universités ont donc occupé et occupent toujours une place essentielle lorsqu'il s'agit de mettre en œuvre évaluations ou travaux de prospective. Dans la suite de cet article, on montrera l'évolution du rôle des universités dans le domaine du conseil, depuis les années soixante. Les exemples qui seront donnés sont généralement choisis parmi les travaux menés par les équipes de recherche auxquels les auteurs appartiennent ou pour leur caractère exemplatif.

\section{Evaluer l'éducation : observer et décrire}

La première période est marquée par des recherches commanditées (c'est-à-dire réalisées à la demande de, et financées par le gouvernement) sans nécessairement de lien direct avec des décisions politiques ou administratives en matière d'éducation. Dans cette configuration, fondée sur la liberté d'enseignement, alors que l'administration de l'enseignement est limitée dans ses actions aux fonctions les plus élémentaires (paye des enseignants, liquidation des subventions...), les chercheurs, bien que dans un cadre de recherche commanditée, réalisent de manière autonome, notamment au sein de l'Association internationale pour l'évaluation du rendement scolaire (IEA) ${ }^{1}$, des études qui semblent avoir très peu d'impact sur les politiques éducatives.

Dans le cadre de ces études internationales, les chercheurs belges francophones, à la suite de Fernand Hotyat et de Gilbert de Landsheere, développent des méthodes de plus en plus sophistiquées et recueillent des ensembles très riches de données qui permettent de mieux comprendre les mécanismes déterminant efficacité et équité des systèmes éducatifs (De Landsheere, 1986 ; Lafontaine et Blondin, 2004).

Ce n'est que dans la seconde moitié des années 1980 que les gouvernements des pays industrialisés commencent à s'intéresser aux travaux menés au sein de l'IEA à des fins de pilotage (Tiana, 2001). Cet intérêt est plus tardif en Belgique. En 1989, celle-ci transfère effectivement ses compétences en matière d'éducation aux Communautés, au sein d'un état fédéral. L'un des problèmes du système éducatif réside alors, comme l'a souligné l'OCDE

\footnotetext{
${ }^{1}$ http://www.iea.nl
} 
(1993), dans l'absence d'évaluations nationales, en dehors des évaluations internationales dont la portée en matière de pilotage reste confidentielle, et même d'objectifs clairs pour son école. Une vaste radioscopie de l'enseignement francophone est alors confiée, mais avec un pilotage très contraignant cette fois, à une équipe interuniversitaire regroupant des chercheurs de quatre universités francophones (Université libre de Bruxelles, Université de Liège, Université catholique de Louvain et Université de Mons-Hainaut). Ce rapport (mars 1992) ne sera jamais publié, mais il permettra de justifier d'importantes mesures d'austérité (Van Haecht, 2004).

Il faut attendre les résultats de la troisième étude sur l'enseignement des mathématiques et des sciences (TIMSS) pour voir réellement les autorités se saisir des résultats et proposer des modifications du curriculum, après une expérimentation contrôlée (Flammang et Forget, 2002). Mais ce sont véritablement les résultats du Programme international pour le suivi des acquis des élèves (PISA, Ocdé), dont la mise en oeuvre est confiée à un service universitaire, qui marque un tournant dans l'utilisation qui peut être faite des résultats des évaluations externes, dès leur publication au début des années 2000 .

En France, interrogeant l'incidence prescriptive des indicateurs internationaux et de leurs usages durant la même période, Agulhon estime qu'ils "participent à la diffusion de normes partagées sur le mode de l'évidence, en particulier sur le rendement de l'investissement éducatif, les acquis des élèves et les niveaux de formation" $(2006$; 322) et influencent la définition des productions statistiques nationales voire, directement ou indirectement, peuvent infléchir des politiques nationales ${ }^{2}$. En Belgique, la participation aux études internationales, l'interprétation de leurs résultats et leur intégration dans la définition de réformes ne semblent perçues ni par le public, ni par le politique - au contraire d'un discours dominant en France, notamment chez les chercheurs ${ }^{3}$ - comme une perte de pouvoir, une mise sous tutelle européenne ou internationale d'un système éducatif national. En Belgique francophone, c'est davantage la succession rapide des réformes que la «délégation politique de l'expertise à des scientifiques » (Normand, 2006, 41) ou l'indépendance de la recherche commanditée qui est soulignée. Cette succession de réformes est notamment pointée par la consultation des enseignants commanditée en 2004, elle aussi, à un service universitaire ${ }^{4}$.

\section{Evaluer le système éducatif pour réguler les politiques}

Les contributions directes des chercheurs à la régulation du système occupent progressivement, dans les années 1990, une place de plus en plus importante, au fur et à mesure du développement de la régulation par l'autorité publique de ce qui jusque-là avait été abandonné à la régulation par le marché. L'absence, en Belgique francophone, d'organes tels que l'Inspection générale de l'Education nationale à qui est dévolu le rôle de «conseil et d'aide à la mise en ceuvre et à l'évaluation de la politique éducative » (Van Zanten, 2004) ou d'une Direction de l'Evaluation et de la Prospective, plaque tournante de l'information qui a aussi bien un rôle de production de statistiques à la demande du politique que de commanditaire de recherches scientifiques (Agulhon, 2006, 321), conduit l'administration ou plutôt le Gouvernement à faire appel aux universités pour réaliser des études, mais aussi contribuer à la mise en œuvre des politiques éducatives.

\footnotetext{
${ }^{2}$ Voir aussi, à ce sujet, Bottani et Vrignaud (2005) et Cytermann et Demeuse (2005).

${ }^{3}$ Voir par exemple, Derouet, 2006.

${ }^{4}$ Les rapports sont téléchargeables à l'adresse :

http://www.enseignement.be/prof/info/ens/consultation2004/consultation2004.asp.
} 
Ainsi, des équipes interdisciplinaires et interuniversitaires sont sollicitées, y compris à travers les textes légaux, par exemple, pour établir l'indice socio-économique utilisé dans le calcul des moyens distribués dans le cadre des politiques de discrimination ou de différenciation positive (Demeuse et Monseur, 1999). L'implication des chercheurs sur ce thème a une histoire. Dans une première phase, les zones d'éducation prioritaires (ZEP) ont été mises en place en Belgique francophone en 1989. Deux rapports (Legiest et al, 1993; Hogge et al, 1995) ont été réalisés par des chercheurs des quatre universités francophones du pays, mais ils sont demeurés non publiés. Ces rapports avaient une visée d'évaluation d'une politique éducative, ils contribuaient donc à l'information de l'administration de l'enseignement, en particulier par l'établissement d'un diagnostic dans un domaine peu couvert par la littérature scientifique (Demeuse, 2002, 93).

En 1998, les ZEP sont remplacées par les discriminations positives. La contribution de la recherche légalement requise dans les décrets du 30 juin 1998 - décret visant à assurer à tous les élèves des chances égales d'émancipation sociale, notamment par la mise en œuvre de discriminations positives - puis du 27 mars 2002, consiste principalement en la détermination de variables à prendre en compte pour établir un indice socio-économique fondamental pour l'attribution des financements des établissements. Le texte prévoit l'approbation du gouvernement sur les variables proposées par les chercheurs. Le caractère interuniversitaire de l'équipe en charge de ce travail, implique la mise en place d'une équipe composée de trois universités ${ }^{5}$. Si le rapport de recherche (non publié) est à destination unique du commanditaire, la recherche donne lieu à une thèse (Demeuse, 2002) et à plusieurs articles.

Cette implication directe des chercheurs se traduit également lors de la mise en place des évaluations externes destinées au pilotage du système, dès leurs débuts en 1994. Le support direct à l'action semble remplacer progressivement le premier alors que le politique prend de plus en plus conscience à la fois du manque d'efficacité et d'équité du système éducatif belge francophone et met en place les outils d'une politique davantage accompagnée (socles de compétences, commission de pilotage, service de statistiques, évaluations externes...).

Si une structure légère de pilotage existe dès 1993 au sein de l'administration, impliquant des chercheurs, mais sans base décrétale, le décret « missions » (1997) institue trois commissions de pilotage examinant les travaux concernant les socles de compétences et les compétences terminales, les programmes et les outils d'évaluation. Ce n'est que le 27 mars 2002 qu'un décret spécifique instaure une commission de pilotage avec des missions précisées. Sa composition - inspecteurs, experts en pédagogie issus des universités et de l'enseignement supérieur non universitaire, représentants des pouvoirs organisateurs, des organisations syndicales, des parents d'élèves et de l'administration - est révélatrice de la répartition des pouvoirs, spécifique à la Belgique francophone.

Cette commission, pour remplir ses missions, confie deux recherches à des équipes interuniversitaires, l'une portant sur la définition d'un système d'indicateurs pour l'enseignement fondamental et secondaire, la seconde proposant une modélisation de l'architecture d'une base de données d'élèves. De nouveau, la construction et même la définition de l'architecture des indicateurs sont confiées aux universités. Si ces recherches demeurent non publiées, leurs résultats sont utilisés, discutés au sein de la commission de pilotage et font l'objet de publications par l'administration.

\footnotetext{
${ }^{5}$ Université de Liège (publique), Université Libre de Bruxelles (libre non confessionnelle) et Université Catholique de Louvain (libre confessionnelle).
} 
Comme en témoignent ces exemples, les contributions des chercheurs à la régulation et à l'évaluation du système éducatif de la Belgique francophone sont à mettre en relation avec « la tendance à un accroissement de l'intervention de l'Etat qui, initialement, jouait un rôle extrêmement modeste dans les questions scolaires» (Maroy et Dupriez, 2000, 80). Pour Maroy et Dupiez, "l'agenda politique met de plus en plus l'accent sur les questions de pilotage, d'administration des systèmes et organisations scolaires » (85). Le gouvernement doit naviguer entre la régulation par un Etat évaluateur (avec l'aide des chercheurs pour la mise en œuvre) visant une meilleure qualité du système, les contraintes des «institutions existantes et les compromis politiques clés fondateurs du système (sur la liberté d'enseignement notamment)»(Maroy, 2005, 16). Ainsi, les résultats produits lors des évaluations externes ne sont publiés qu'au niveau global du système (et non des réseaux ou des établissements) afin de ne pas accroître la logique de quasi-marché scolaire pointée comme à l'origine des fortes inégalités du système éducatif. La portée des évaluations externes et leurs impacts en termes de régulation et de pilotage sont donc limités à un pilotage global et non à une régulation au niveau des établissements d'enseignement.

Les politiques éducatives évoluent progressivement vers une approche qu'il serait possible de qualifier d'evidence-based policies, au sens de Lessard, c'est-à-dire qui s'appuient "le plus possible, voire découl[ant] directement de la "recherche" de qualité, et non pas de $l$ ' " idéologie ». » $(2006,26)$. Cela se traduit notamment, dans les documents officiels, comme le Contrat Stratégique pour l'Education, publié en janvier 2005 par le Gouvernement, par des mentions explicites à des résultats de recherche alors même que ce texte, comme son nom l'indique, est un document d'orientation. Naturellement, ces mentions explicites n'excluent pas les prises de position politiques mais aussi, comme l'indiquent les travaux de Draelants (2006) sur l'évolution du premier degré de l'enseignement secondaire entre 1994 et 2004, une «montée de la légitimation par les savoirs de terrain » se traduisant au niveau des politiques par une recherche du consentement des enseignants et de leurs syndicats.

\section{Conseiller le politique : émergence de la prospective en éducation}

La prospective en éducation, est caractérisée par la recherche de «ce qui pourrait marcher ». L'implication des chercheurs dans l'outillage, voire la définition, de politiques à venir constitue la troisième approche. Celle-ci est contemporaine d'un changement important dans le domaine des politiques éducatives : quittant son approche exclusivement "comptable ", l'administration scolaire s'oriente vers une approche davantage orientée vers les résultats et les objectifs à moyen terme, comme l'indiquent le Contrat Stratégique pour l'Education (2005) et son successeur, le Contrat pour l'Ecole (2005).

Deux études s'inscrivent clairement dans ce troisième paradigme. La première, dont le rapport est intitulé «Les bassins scolaires : de l'idée au projet », a été consacrée à la mise en place de politiques territorialisées pour lutter contre les fortes inégalités scolaires (Delvaux et al, 2005). La seconde, avec des objectifs semblables énoncés sous le slogan « Non aux écoles ghettos » dans le Contrat pour l'Ecole qui prévoit explicitement sa réalisation, explore des modes de financement des établissements scolaires tenant davantage en compte les caractéristiques socio-économiques des élèves (Demeuse et al, 2007). 
Inscrite dans un contexte marqué par de forts débats autour de l'orientation de la politique scolaire (Delvaux et al., 2005), la première étude s'appuie sur un engagement politique clair ${ }^{6}$ : travailler à la réduction des inégalités aussi bien entre élèves qu'entre écoles. Comme lors de la mise en place des discriminations positives à la fin des années 1990, l'équipe en charge de cette recherche est à la fois interuniversitaire, regroupant quatre universités francophones, et interdisciplinaire puisque psychopédagogues, sociologues et géographes sont associés. L'équipe reçoit "carte blanche» du gouvernement pour proposer des formules innovantes (des scénarios); elle ne peut cependant faire fi des réalités du contexte belge francophone. Cette recherche se démarque principalement par sa caractéristique de prospective où l'investigation des dimensions visées par la commande et les argumentations scientifiques débouchent sur des scénarisations contrastées, mais réalistes. Aucun de ces scénarios ne satisfait tous les critères retenus, en particulier ceux d'autonomie, d'efficacité et de respect des délimitations institutionnelles existantes. Il s'agit d'une véritable prise de risque des scientifiques dont les propositions s'appuient sur une évaluation de l'existant, un repérage de variables clés qui sont mises en interaction au sein de scénarios, laissant naturellement les choix et les décisions stratégiques au politique. Le rapport, non diffusé par le Gouvernement pendant près de 16 mois $^{7}$, a finalement fait l'objet d'une communication, dans le cadre d'un colloque réunissant politiques, chercheurs et acteurs de l'éducation. Celle-ci a fait l'objet d'une forte médiatisation, notamment parce que certains scénarios jugés les plus efficaces par rapport aux objectifs de démocratisation visés peuvent être considérés comme remettant en cause la liberté d'enseignement.

La seconde recherche de type prospectif que nous prenons en exemple a fait l'objet d'une médiatisation avant même sa conclusion. En effet, une recherche sur le (re)financement des écoles a été annoncée à plusieurs reprises par la ministre en charge de l'enseignement. Cette étude, également menée par une équipe interuniversitaire, s'attache à proposer des formules de financement alternatives des établissements, en y incluant notamment comme paramètre l'origine socio-économique des élèves. Après un état des lieux du système éducatif et l'identification d'indicateurs pertinents, diverses simulations de financement possibles, prenant en compte différentes réalités du public accueilli par les écoles, sont élaborées. L'idée centrale est de permettre aux décideurs politiques, à l'aide d'un simulateur, d'envisager - tant au niveau d'une école donnée que du budget total - l'impact de différents scénarios de financement. Dans cette étude, comme dans la précédente, la même modalité de conseil est mise en œuvre : les chercheurs élaborent et argumentent des scénarios d'évolution du système éducatif en fonction de la décision que les politiques choisiraient de prendre. Il ne s'agit donc pas d'élaborer des recommandations qui définiraient une politique, mais bien d'outiller la prise de décision en, d'une part, proposant des scénarios, y compris à travers un simulateur de financement, s'inscrivant dans les objectifs définis par le Contrat pour l'Ecole et, d'autre part, en anticipant les effets possibles de ces options.

\section{Conclusion}

\footnotetext{
6 Une déclaration politique communautaire, en juillet 2004, puis une déclaration commune entre le Gouvernement de la Communauté française, les organisations représentatives de la Communauté éducative et les partenaires sociaux en novembre 2004 ont précédé la signature du contrat pour la recherche en décembre 2004.

Si les projets de recherche font l'objet d'un appel d'offre, le commanditaire ne s'engage pas à rendre le rapport public, par exemple via le site du Gouvernement. Le commanditaire reste libre de disposer des résultats de l'étude. Les chercheurs doivent notifier leur intention de publier des informations en rapport avec le sujet de la recherche subventionnée à la Ministre, celle-ci pouvant faire valoir observations et objectifs dans un délai de trente jours calendrier. Les publications doivent mentionner que l'étude est à l'initiative du Ministre.
} 
Si des sociologues français (notamment, Poupeau, 2003, Agulhon, 2006) questionnent l'indépendance d'une recherche scientifique dont les objectifs sont définis par un appel d'offre émanant du politique à travers l'administration, ils soulignent également l'interaction entre recherche et politique. D'une part, des problématiques sont mises au jour par les chercheurs et influencent l'institution scolaire et, d'autre part, les chercheurs peuvent être perméables à l'air du temps, aux attentes sociales, dans la définition et la menée de leurs travaux. Dans un cadre proche - la science des politiques publiques d'éducation -, Derouet pointe la fragilité aux plans épistémologiques et politiques de travaux dans lesquels "les complicités entre les chercheurs et la haute administration sont souvent telles que la recherche peut être soupçonnée de n'être qu'une mise en forme de l'opinion commune de la hiérarchie » $(2000,7)$.

Pour sa part, Dutercq (2000), questionne le rôle de l'expert - chercheur ou consultant privé dans ses actions d'évaluation, en particulier concernant son pouvoir. Ainsi, toute évaluation comporte une phase d'interprétation au regard d'un référentiel - de références : "Toute expertise est interprétation, toute interprétation s'élabore au nom de références, d'un idéal, parfois d'intérêts » $(2000,147)$. En plus d'une dépendance éventuelle à un commanditaire, c'est le manque d'explicitation des référentiels que pointe Dutercq. Les interprétations des évaluations risquent d'être comprises comme des faits ou des preuves alors qu'elles devraient être des repères destinés «à éclairer l'action des responsables et à fournir des instruments de pilotage » (2000, 147). Dans le cadre du conseil, l'évaluation des politiques éducatives ne devrait pas doter le chercheur d'un pouvoir décisionnel, qu'il soit usurpé par le chercheur comme abandonné par le politique.

Pourtant, l'opposition entre un risque de compromission du chercheur et sa toute-puissance sur la décision politique ne sont-ils pas dépassés dans le débat épistémologique actuel? Derouet note à ce propos que «ce n'est que très récemment que les sciences humaines et sociales sont sorties de l'alternative simpliste entre une visée utilitaire qui les met au service des puissants et le superbe isolement d'une science critique qui a les mains propres mais qui n'a pas de mains » $(2000,8)$.

En Belgique francophone, la majorité des équipes scientifiques semble concevoir la coopération entre science et administration comme «correspondant à une mission, partie intégrante de la notion de service public [même si les universités ne relèvent pas directement de la fonction publique] : mettre les résultats de la recherche au service des acteurs pour les aider à renouveler ce que Lucien Fèbvre appelait leur " outillage mental » 》 (Derouet, 2000, 8). Il s'agit ici aussi bien des acteurs praticiens de l'éducation que des acteurs décideurs de l'éducation.

S'appuyant sur les travaux de Pelletier $(2001)$, Dupriez $(2004,12)$ rappelle les trois sources de légitimité, potentiellement concurrentes, à l'œuvre dans le monde scolaire :

- la légitimité démocratique "entoure toute prescription émanant des autorités politiques $»$ :

- la légitimité de marché correspond à «ce qui est susceptible d'emporter l'adhésion des parents-consommateurs d'école »;

- l'expertise professionnelle des enseignants et des personnels de l'éducation.

Dans ce propos, aucune référence n'est faite à une légitimité ou à une expertise scientifique. Le caractère scientifique ou la validité des propositions issues des évaluations ne constituent donc pas, dans la description que Dupriez donne du monde de l'éducation belge francophone, des conditions suffisantes pour définir une légitimité scientifique. 
Nous avons cependant montré en quoi la recherche, dans ses relations avec l'administration scolaire, se situe dans l'outillage et le conseil, en particulier grâce au recours aux modélisations et à la scénarisation prospective. Les caractères interuniversitaire, pluridisciplinaire et non centré sur une personnalité scientifique médiatique sont également des garanties d'un débat démocratique possible où les chercheurs fournissent une part de l'argumentation sans pour autant préjuger des décisions à prendre.

Les deux premières périodes du développement de la recherche en administration scolaire ont permis d'éclairer la manière dont les décideurs politiques pensent les problèmes. Ainsi, la sensibilisation à de nouveaux enjeux éducatifs, la conceptualisation des problèmes rencontrés comme l'évaluation des politiques menées ont débouché progressivement sur la définition d'une ligne politique affirmée (Contrat stratégique pour l'Education et Contrat pour l'Ecole). L'intérêt des décideurs politiques pour les résultats de recherches en éducation s'est alors déplacée de la description d'une situation vers une démarche prospective ayant pour but d'anticiper des changements futurs, de donner des conseils à propos de différentes alternatives, et de stimuler des perspectives innovantes (Ross et al., 2006).

\section{Bibliographie :}

AGULHON, C., (2006), La mesure en éducation, un outil au service du politique. L'exemple de la production française, L'Orientation Scolaire et Professionnelle, 35, 3, 315-336.

BOTTANI, N., VRIGNAUD, P., (2005), La France et les évaluations internationales. Rapport établi à la demande du Haut Conseil l'évaluation de l'école $n^{\circ} 16$, Paris, Haut Conseil de l'évaluation de l'école.

CYTERMANN, J.R.., DEMEUSE, M., (2005), La lecture des indicateurs internationaux en France. Rapport établi à la demande du Haut Conseil l'évaluation de l'école $n^{\circ} 18$, Paris, Haut Conseil de l'évaluation de l'école.

DE LANDSHEERE, G. (1986). La recherche en éducation dans le monde. Paris : Presses universitaires de France, Coll. « Pédagogie d'aujourd'hui ».

DELVAUX, B., DEMEUSE, M., DUPRIEZ, V., FAGNANT, A., GUISSET, C., LAFONTAINE, D., MARISSAL, P., MAROY, C., (2005), Les bassins scolaires : de l'idée au projet. Propositions relatives aux domaines d'intervention, aux instances et aux territoires, Rapport à la Communauté française de l'étude intitulée «Définition, cartographie, rôles et modes de régulations des bassins scolaires ».

DEMEUSE, M., (2002), Analyse critique des fondements de l'attribution des moyens destinés à la politique de discrimination positive en matière d'enseignement en Communauté française de Belgique, Dissertation doctorale en sciences psychologiques (Université de Liège).

DEMEUSE, M., MONSEUR, C., (1999), Analyse critique des indicateurs déterminant l'attribution des moyens destinés à la politique de discrimination positive en Communauté française de Belgique, Mesure et Evaluation en Education, 22(2-3), 97-127. 
DEMEUSE, M., DEROBERTMASURE, A., FRIANT, N., HERREMANS, T., MONSEUR, C., UYTTENDAELE, S., VERDALE, N., (2007), Etude exploratoire sur la mise en cuvre de nouvelles mesures visant à lutter contre les phénomènes de ségrégation scolaire et d'inéquité au sein du système éducatif de la Communauté française de Belgique, Bruxelles : Rapport de recherche non publié.

DEROUET, J.L., (2000), Une science de l'administration scolaire est-elle possible ? Revue Française de Pédagogie, 130. 5-14.

DEROUET, J.L., (2006), Entre la récupération des savoirs critiques et la construction des standards du management libéral : bougés, glissements et déplacements dans la circulation des savoirs entre recherche, administration et politique en France de 1975 à 2005, Revue Française de Pédagogie, 154, 5-18.

DRAELANTS, H., (2006), Politiques d'éducation et changement institutionnel. Le cas de la réforme du premier degré de l'enseignement secondaire en Belgique francophone, Thèse de doctorat en sociologie, Université Catholique de Louvain.

DUPRIEZ, V., (2004), La place de l'évaluation comme ressource pour le pilotage des systèmes scolaires : état des lieux en Belgique francophone et en Angleterre, Les Cahiers de Recherche en Education et Formation, 35.

DUTERCQ, Y., (2000), Administration de l'éducation: nouveau contexte, nouvelles perspectives, Revue Française de Pédagogie, 130, 143-170.

FLAMMANG, C., FORGET, A., (2002), Amélioration de l'enseignement des sciences au premier degré de l'enseignement secondaire. Analyse des effets à court et à moyen terme e l'introduction d'énigmes scientifiques dans les classes, Cahiers du Service de Pédagogie Expérimentale, 9-10, 131-167.

HOGGE, B., DE VLEESCHOWER, C. WATERSCHOOT, V., BLONDIN, C., MANÇO, A., CHAVEPEYER, I., CLAUS, S., (1995), Evaluation des Zones d'Éducation Prioritaires en Wallonie : Moyens mis en cuvre et stratégies, Bruxelles, Communauté française de Belgique, Ministère de l'Éducation, de la Recherche et de la Formation. Commission «Zones d'Éducation Prioritaires ».

HUBIN, J.-P., (2005), La commission de pilotage du système éducatif. Tables Rondes, 2, 18 , Document accessible à l'adresse http://www.enseignement.be/copi/tablesrondes_sup200505.pdf, consulté en février 2007.

LAFONTAINE, D., BLONDIN, C., (2004), Regards sur les résultats des élèves en Communauté française. Apports des enquêtes de l'I.E.A., de PISA et des évaluations externes, Bruxelles, De Boeck.

LEGIEST, N., POHL, S., BLONDIN, C., LAFONTAINE, D., HOGGE, B., HOUX, M., (1993), Évaluation des premières zones d'éducation prioritaires en Communauté française (Zones 2 et 3 : Bruxelles, Bruxelles, rapport non publié.

LESSARD, C., (2006), Le débat américain sur la certification des enseignants et le piège d'une politique éducative evidence-based, Revue Française de Pédagogie, 154, 19-31. 
MAROY, C., DUPRIEZ V., (2000), La régulation dans les systèmes scolaires. Proposition théorique et analyse du cadre structurel en Belgique francophone, Revue Française de Pédagogie, 130, 73-87.

MAROY, C., (2005), Vers une régulation post-bureaucratique des systèmes d'enseignement en Europe, Les Cahiers de Recherche en Education et Formation, 49.

MINISTÈRE DE LA COMMUNAUTÉ FRANÇAISE DE BELGIQUE \& ETNIC, (2006), Les indicateurs de l'enseignement. Document accessible à l'adresse :

http://www.enseignement.be/prof/dossiers/indicateurs/Indic200612 TOUT.pdf , consulté en février 2007.

NORMAND, R., (2006), L'école efficace ou l'horizon du monde comme laboratoire, Revue Française de Pédagogie, 154, 33-43.

OCDE, (1993), Examens des politiques nationales d'éducation. Belgique, Paris, OCDE.

Pourquoi les enseignants sont-ils en froid avec les réformes pédagogiques ? (2007), AlterEduc, 139, 9 février 2007.

PELLETIER, G., (2001), Décentralisation, régulation et gouvernance des systèmes éducatifs : un cadre de référence. In G. Pelletier (Ed.), Autonomie et décentralisation en éducation : entre projet et évaluation, Montréal, Editions de l'AFIDES.

Ross, K.N., Jürgens Genevois, I., (Eds.), (2006), Cross-national studies of the quality of education : planning their design and managing their impact, Paris, UNESCO - IIPE.

TIANA, A., (2001), Le monde comme laboratoire éducatif, Politiques d'Education et de Formation. Analyses et Comparaisons Internationales, 3, 47-57.

VAN HAECHT, A., (2004), Histoire et critique en sociologie de l'éducation : le cas de la Communauté française de Belgique, Education et Sociétés, 13(1), 119-140.

VAN ZANTEN, A., (2004), Les politiques éducatives entre le dire et le faire, Sciences Humaines, 153. 